\title{
Relaciones y afinidades biológicas de la población prehistórica de San Pedro de Atacama
}

José A. Cocilovo ${ }^{1}$, Silvia Quevedo ${ }^{2}$ y Francisco RothHammer ${ }^{3}$

\section{Introducción}

La preocupación por el estudio de las características biológicas de la población prehistórica en San Pedro de Atacama surge en la década del 60 con el progreso de los trabajos arqueológicos realizados en la zona (Le Paige 1957, 1958, 1961, 1963, 1964; Orellana 1961, 1962, 1963, 1964, etc.) y con ellos la integración de una importante colección de restos óseos hasta hoy escasamente descrita a pesar de la existencia de varios trabajos realizados con sus materiales, entre los que se destacan los de Larraín del Campo (1961), Le Paige (1961 y 1966), Munizaga (1964, 1969 y 1974) y Henckel (1968a, 1968b).

En 1970, el Instituto de Antropología de Córdoba, con la participación de uno de los autores (J.A.C.) y la dirección de A. J. Marcellino, llevó a cabo el relevamiento de 82 piezas craneales de las colecciones del Museo de San Pedro de Atacama, cuyos resultados han permanecido inéditos hasta la fecha.

A pesar de la importancia antropológica de la población prehistórica de San Pedro de Atacama, no existen en la literatura científica trabajos realmente de envergadura que permitan una adecuada caracterización de la misma, y con ello el establecimiento de su origen, desarrollo y vinculaciones con otras áreas latinoamericanas. Tampoco hasta el momento se conoce la existencia de proyecto alguno interesado en la realización de un estudio sistemático desde el punto de vista biológico.

La existencia de algunos trabajos inéditos y otros publicados que se ocupan de la población de San Pedro de Atacama, y la necesidad de aportar alguna información de interés o de adelantar simples

1 Universidad Nacional Río Cuarto, Río Cuarto, ARGENTINA.

2 Museo Nacional de Historia Natural, Santiago, CHILE.

3 Departamento de Biología Celular y Genética, Facultad de Medicina, Universidad de Chile, Santiago, CHILE. hipótesis sobre sus relaciones de parentesco, estimuló la realización del presente que a modo de informe preliminar se ofrece en esta oportunidad. Además, el carácter de la mesa redonda correspondiente al Simposio de Arqueología Atacameña, celebrado entre el 10 y 15 de enero de 1983, nos indujo a encarar su exposición con un cierto estilo y un trasfondo metodológico consecuentes y, a la vez apropiados, con el momento por el cual transita la disciplina en nuestro país.

\section{Primera aproximación}

En antropología prehistórica las relaciones de parentesco entre las poblaciones pueden ser inferidas a partir de su caracterización biológica y del análisis comparativo de las semejanzas y diferencias entre dos o más grupos. Con respecto a este punto, podría ensayarse la confección de un gráfico de dos coordenadas, si disponemos de los valores medios de dos variables métricas, por ejemplo, correspondientes a un conjunto de poblaciones sudamericanas. La fuente más expedita para obtener esta información es el clásico trabajo de Stewart y Newman (1950) en donde se presentan un conjunto de tablas con los valores medios, separados por sexo, de variables craneométricas seleccionadas por su aparente independencia de la deformación artificial.

Por ejemplo, podemos tomar en cuenta la anchura bicigomática y el diámetro nasión-prostión y consignar en el mencionado gráfico los valores medios para cada grupo (Gráfico 1). Al final de la operación estos quedarán representados por puntos distribuidos en el plano con distancias variables entre sí. Nuestro grupo problema, en este caso la población prehistórica de San Pedro de Atacama, puede ser ubicado de la misma manera, al definir en dicho plano un punto caracterizado por la intersección de las coordenadas respectivas. Así la comparación puede ser realizada e inferidas las relaciones entre nuestro grupo y los restantes, intuitivamente, a partir de la evaluación personal de las distancias entre los puntos. 


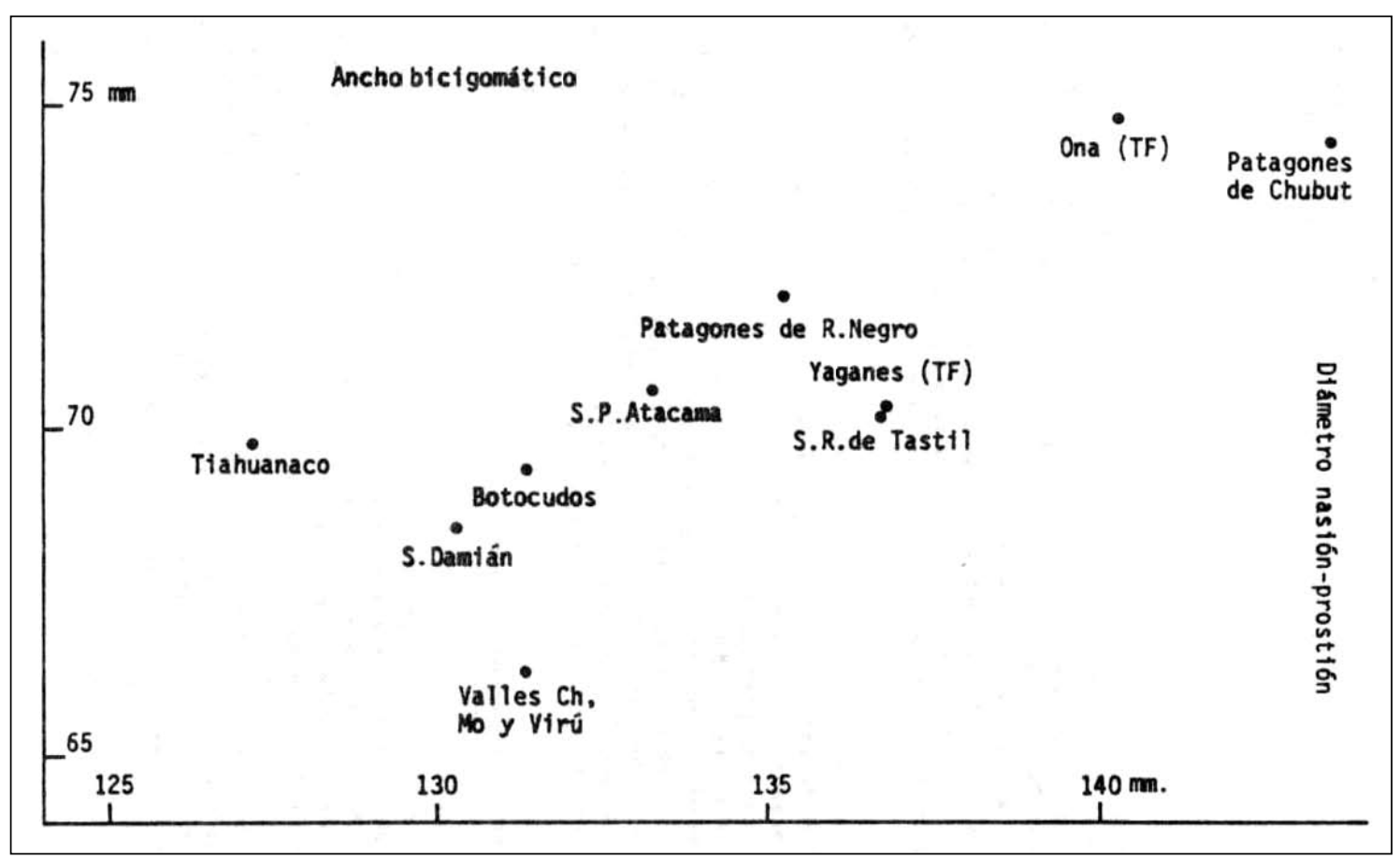

Gráfico 1. Ancho bicigomático y diámetro nasión-prostión.

Así, observamos que si tenemos en cuenta las dimensiones de la cara, San Pedro de Atacama se encuentra próximo a Botocudos, Santa Rosa de Tastil y Patagones de Río Negro. A partir de esta información podríamos imaginarnos ricas explicaciones sobre las vinculaciones, parentesco, el origen y el desarrollo del grupo en estudio. Pero nada nos impide continuar con nuestra operación y ensayar el comportamiento de los puntos medios en el plano, considerando de a pares cualquier número y combinación de nuevas variables. Si ensayamos las dimensiones de la nariz, San Pedro de Atacama aparece más próximo a Patagones de Río Negro; si intentamos realizar la comparación con el índice orbitario y el índice de altura media de Hrdlicka, ahora, debemos agregar el grupo de Araucanos en nuestro esquema anterior de relaciones. Luego podríamos combinar el índice facial superior y el índice orbitario, lo cual nos llevaría seguramente a establecer la vinculación entre San Pedro y el grupo de Río Negro mencionado con el de Patagones de Chubut. Si seguimos nuestro viaje imaginario por la misma dirección, comprobaremos al final la desazón provocada por el descubrimiento de un impreciso e incalculable margen de incertidumbre, pero no menos real ni menos evidente, asociado con cada una de las hipótesis antes derivadas. Sobre nuestra mesa de trabajo seguramente se habrán amontonado una veintena de gráficos similares al primero aquí presentado, como resultado de toda una jornada de febril actividad, en la búsqueda de un ideal casi platónico, basado en la identificación absoluta para establecer el origen y la distancia más pequeña para imaginar contactos o relaciones biológicas de parentesco, con lo cual, sin advertirlo, nuestras hermosas conclusiones se transforman en un verdadero despropósito desde el punto de vista científico.

Igual nos sucedería si en lugar de ensayar una aproximación gráfica tratamos de inferir relaciones biológicas entre las poblaciones, por la sencilla comparación aritmética de sus valores medios, para cualquier conjunto de variables consideradas individualmente. Las verdaderas relaciones de parentesco serán difíciles de establecer por la indefinición del verdadero valor y sentido de las diferencias o distancias encontradas entre los grupos estudiados.

Felizmente, una suerte de recomposición teórica y metodológica surgida con el progreso mismo de la disciplina, con la colaboración de la genética de poblaciones y con el desarrollo de potentes técnicas 
de experimentación numérica, posibilitó la consecución de los objetivos antes enunciados a través de sucesivas aproximaciones en experiencias repetibles, observaciones verificables y conclusiones válidas desde el punto de vista científico.

\section{Segunda aproximación}

Con la idea de aportar información sobre la influencia del espaciamiento geográfico en la conformación biológica de un conjunto de poblaciones latinoamericanas, fueron realizados varios ensayos hasta 1976 y publicados recién en 1981 (Cocilovo 1981).

Con la aplicación de técnicas estadísticas de análisis multivariado, se logró una mayor eficiencia en la recuperación de la información osteológica contenida en series éditas e inéditas de Perú, Bolivia, norte de Chile, Noroeste Argentino, litoral fluvial, Patagonia y Tierra del Fuego.

La organización de la subespecie en unidades discretas llamadas poblaciones, la diferenciación morfológica y su correlación con las distancias geográficas, así como la posibilidad de hacer las primeras inferencias sobre las relaciones de parentesco, fueron las principales conclusiones derivadas del citado trabajo. Hoy releyendo el mismo es posible extraer algunas observaciones sobre las relaciones y afinidades biológicas de San Pedro de Atacama y otras poblaciones prehistóricas.

En efecto, si observamos la matriz de distancias biológicas basada en el cálculo de la $\mathrm{D}^{2}$ de Mahalanobis para nueve variables esplacnocraneanas, lograda en el citado trabajo, San Pedro de Atacama se presenta asociada más estrechamente con las poblaciones de Santa Rosa de Tastil y Valles Calchaquíes del noroeste Argentino. Un arreglo tipo análisis de conglomerados (cluster analysis) confirmó dicha apreciación revelando la integración de un subgrupo andino bastante diferenciado del conglomerado continental, formado primeramente por San Pedro y Santa Rosa y luego por valles calchaquíes.

Son coherentes también las observaciones derivadas de la distribución de las poblaciones en el espacio reducido por medio de las funciones discriminantes. El cuadro de la reclasificación de la muestra de San Pedro de Atacama, atendiendo a la mayor probabilidad de cada individuo de pertenecer a cualquiera de los doce grupos considerados, indica una alta posibilidad de intercambio con valles calchaquíes y Santa Rosa de Tastil (Cocilovo 1981: Cuadros 2 y 4, Figs. 2, 3 y 4 ).

\section{Tercera aproximación}

Desde el año 1979, se puso en marcha el programa de Microevolución en Poblaciones Prehistóricas del Area Andina, auspiciado por la Universidad de Chile, el Museo Nacional de Historia Natural y la Universidad Nacional de Río Cuarto. Su desarrollo exigió la incorporación de nuevos datos del valle de Azapa, Camarones, Pisagua, etc., y el ensayo de nuevas y más eficientes concepciones teóricas derivadas de la genética de poblaciones, acompañadas por importantes técnicas de experimentación numérica (Rothhammer et al. 1981, 1982 y Ms), que permitieron estudiar la extinción del parentesco con la distancia geográfica, y por primera vez establecer su comportamiento en función del tiempo.

Entre los múltiples ensayos realizados hasta la fecha las afinidades y relaciones biológicas de San Pedro de Atacama aparecen como un subproducto necesario resultante de la comparación de cerca de 24 grupos sudamericanos prehistóricos, tomando en cuenta simultáneamente las mismas variables esplacnocraneanas.

Así, disponiendo de una matriz de varianzas y covarianzas común calculada independientemente por muestreo por el excesivo número de observaciones, y de los vectores medios de 24 poblaciones fue posible obtener una nueva matriz de $\mathrm{D}^{2}$, en la cual se ponen de manifiesto las relaciones de parentesco entre los grupos considerados de Perú, Bolivia, Chile, Argentina y Tierra del Fuego. Con la ayuda de una técnica de análisis de conglomerados (cluster analysis) dichos grupos fueron ordenados en un diagrama dendrítico para facilitar la interpretación de la citada matriz de distancias.

Las observaciones realizadas en la segunda aproximación fueron plenamente confirmadas en esta oportunidad, y al propio tiempo, con la ampliación de los términos de comparación, se han descubierto nuevas e interesantes relaciones.

La población de San Pedro de Atacama estrechamente asociada con la de Santa Rosa de Tastil integra un amplio conglomerado andino formado además por Paucarcancha y Tiahuanaco, la Isla y el Pucara de Tilcara, valles calchaquíes y Belén, y una serie de araucanos del sur de Chile. 


\section{Conclusiones}

De acuerdo con la evidencia disponible es posible afirmar que la población prehistórica de San Pedro de Atacama, considerada globalmente, parece haber participado de un fondo biológico común con otros grupos del Noroeste Argentino y el sur de Chile, de establecimiento y desarrollo contemporáneo. La estrecha asociación con Santa Rosa de Tastil indicaría un activo flujo genético entre ambas poblaciones, las cuales se integran en un subsistema biológico formado por valles calchaquíes, Belén y araucanos. En el esquema taxonómico obtenido con la ayuda del análisis de conglomerados, la presencia de Paucarcancha y de Tiahuanaco, aunque integrados a distancias mayores coopera para definir en el conjunto de poblaciones continentales un tronco andino de características peculiares y seguramente con una historia biológica común, diferente de los acontecimientos etnodinámicos operados en el valle de Azapa, costa norte de Chile, Norte Semiárido, litoral y Patagonia argentina. Resulta por demás significativa la existencia comprobada de un conjunto insular, aislado de los anteriores, formado por los grupos yagan y alacaluf, asociados con ona.

Siguiendo con nuestro estilo de aproximaciones sucesivas, la inspección de la matriz de $\mathrm{D}^{2}$ también revela que las distancias biológicas transversales entre las poblaciones del Noroeste Argentino y el Norte Semiárido de Chile son mayores que las calculadas con respecto a San Pedro de Atacama, pareciendo indicar con ello una vía de intercambio entre ambas regiones a través del Desierto de Atacama.

Lamentablemente, aun el registro bioantropológico es sumamente fragmentario para elaborar conclusiones definitivas, y la información cultural y cronológica asociada con algunas colecciones osteológicas poco ayuda en la tarea de reconstrucción. Sin embargo, confiamos que la incorporación de nuevos materiales inéditos tanto del norte de Chile como de Puna y Quebrada de Humahuaca nos posibilitará en un futuro próximo, junto con un necesario estudio de periodificación biológica en el oasis de San Pedro de Atacama, alcanzar una explicación más científica sobre el origen y desarrollo de la población local. Esto último será factible cuando dispongamos de estudios más detallados sobre los distintos componentes culturales y cronológicos y su vinculación con grupos afincados en regiones vecinas. ${ }^{4}$

Agradecimientos Este estudio se financió parcialmente a través del proyecto B518845 del Depto. de Desarrollo de la Investigación (Dirección General Académica de la Universidad de Chile) y de los proyectos Nos 1068 y 1174 del Fondo Nacional de Desarrollo Científico y Tecnológico.

\section{REFERENCIAS CITADAS}

COCILOVO, J. A., 1981. Estudio sobre discriminación y clasificación de poblaciones prehispánicas del Noroeste Argentino. Publicación Ocasional del Museo Nacional de Historia Natural 36.

HENCKEL, C., 1966a. Estudios de cráneos de San Pedro de Atacama y observaciones acerca de la deformación craneana. Boletín de la Sociedad de Biología Concepción 39: 33-48.

— 1966b. Cráneos de San Pedro de Atacama. 36 Congreso Internacional de Americanistas vol. 2, pp. 339-350. Sevilla.

LARRAIN DEL CAMPO, R., 1961. Protocolos craneométricos. Contexto y estudio anatómico de 27 cráneos de la colección del Museo Arqueológico de San Pedro de Atacama. Anales de la Universidad del Norte 1 (1): 37-96.

LE PAIGE, G., 1957. Descubrimientos en San Pedro de Atacama, Mensaje 56: 14-17 y 28.

1958. Antiguas culturas atacameñas en la cordillera chilena. Anales de la Universidad Católica de Valparaíso 4-5: 15-143.
_ 1961. Estudios craneométricos de la colección del Museo Arqueológico de San Pedro de Atacama. Anales de la Universidad del Norte 1: 12-35.

— 1963. Continuidad o discontinuidad de la cultura atacameña. Congreso Internacional de Arqueología de San Pedro de Atacama, Anales de la Universidad del Norte 2: 5-25.

_ 1964. El Precerámico en la cordillera atacameña y los cementerios del Período Agroalfarero de San Pedro de Atacama. Anales de la Universidad del Norte 3: 1-277.

MUNIZAGA, J., 1964. Comparaciones de poblaciones precolombinas del norte de Chile. Antropología 2: 87-95.

4 Nota de la edición original: La creación del Instituto de Investigaciones Arqueológicas R. P. Gustavo Le Paige s. j. (Universidad del Norte) ha asumido esta situación, creando un Laboratorio de Antropología Física, que abordará de manera interdisciplinaria tal problemática. 
1969. Deformación craneana intencional en San Pedro de Atacama, Actas V Congreso Nacional de Arqueología Chilena, pp. 129-134.

1974. Deformación craneal y momificación en Chile. Antropología, Universidad Nacional Autónoma de México 11: 329-336.

- 1974. Paleopatología chilena (informe preliminar). Antropología Nueva Epoca 1: 35-39.

ORELLANA, M., 1961. Acerca de la arqueología del Desierto de Atacama. Boletín de la Universidad de Chile 27: 41-43.

- 1962. Recientes investigaciones arqueológicas en San Pedro de Atacama. Mensaje 108: 155-160.

1963. La Cultura San Pedro. Estudios Antropológicos 17: 3-43.

1964. Acerca de la cronología del Complejo Cultural San Pedro de Atacama. Antropología 2: 96-104.
ROTHHAMMER, F., S. QUEVEDO, J. A. COCILOVO, G. FOCACCI y E. LLOP, 1981. Microevolución en poblaciones prehistóricas del Area Andina II. Variación craneométrica cronológica en los valles de Arica. Chungara 8: 275-280.

ROTHHAMMER, F., J. A. COCILOVO, S. QUEVEDO y E. LLOP, 1982. Microevolution in prehistoric Andean population I. Chronologic craneometric variation. American Journal of Physical Anthropology 58: 391-396.

ROTHHAMMER, F., S. QUEVEDO, J. A. COCILOVO y E. LLOP, Ms. Microevolution in prehistoric Andean population. IV. Chronologic non-metrical cranial variation.

STEWART, T. D y M. T. NEWMAN, 1950. Anthropometry of South American indian skeletal remains. Handbook of South American Indians 6: 19-42, Smithsonian Institution, Bulletin 143, Washington D. C. 